\title{
Model-free control for VSC-based HVDC systems
}

\author{
Abderrahmane BARKAT*, Bogdan MARINESCU*, Cédric JOIN ${ }^{\dagger} \uparrow \|$ and Michel FLIESS $\ddagger \rrbracket$ \\ * École Centrale Nantes-LS2N-CNRS, 1 rue de la Noë, 44321 Nantes, France \\ Abderrahmane.Barkat@12s.centralesupelec.fr, Bogdan.Marinescu@ec-nantes.fr \\ † CRAN (CNRS, UMR 7039), Université de Lorraine, BP 239, 54506 Vandœuvre-lès-Nancy, France \\ Cedric.Join@univ-lorraine.fr \\ ‡ LIX (CNRS, UMR 7161), École polytechnique, 91128 Palaiseau, France \\ Michel.Fliess@polytechnique.edu \\ IAL.I.E.N., 7 rue Maurice Barrès, 54330 Vézelise, France. \\ \{cedric.join, michel.fliess\}@alien-sas.com \\ ${ }$ Projet Non-A, INRIA Lille - Nord-Europe, France.
}

\begin{abstract}
The High-Voltage Direct Current (HVDC) transmission lines are power electronics based elements more and more used in power grids. In addition to the transport capacity, they provide more control degrees of freedom and possibilities than the classic AC lines. As a consequence, the way in which these controls are synthesized has an impact not only on the dynamics of HVDC power converters but also on the ones of the neighbor AC zone of the HVDC link. To ensure optimal impact, the dynamics which involve neighbor elements have to be taken into account at the control stage. In a standard model-based control, this often leads to complex control models. The model-free control is a recently developed approach, with several successful applications. It uses a very simplified "ultra-local" model, which is continuously data-driven adapted. This approach is employed here for a HVDC in the AC grid context mentioned above. The performances and robustness are compared with the ones of the classic vector-control.
\end{abstract}

Index Terms - High Voltage Direct Current (HVDC), Voltage Source Converter (VSC), model-free control, intelligent controllers.

\section{INTRODUCTION}

Modern power grids contain more and more complex dynamic elements, most of the times based on power electronics. This is the case of the HVDC links. They are also active elements which have an impact on the neighborhood of the AC network in which they are inserted. Particularly, HVDCs controls may affect the transient stability of the near zone (see, e.g., [1]). This impact was optimized in [2] and [3] by using an extended control model. However, as this model captures several grid dynamics in addition to the ones of the HVDC link, its order may be high. This situation is encountered in many other power systems applications. For example, analysis and damping of inter-area modes of a grid needs a model of the whole synchronous zone which results in high-order mathematical objects. Another typical example is the so-called secondary regulations (voltage and frequency) for which a global vision of the system is also necessary.

In order to avoid these difficulties related to the size of the control model, a recently developed methodology called "model-free control" [4], [5] is used here. Indeed, this approach does not need a large-scale precise mathematical model since the system is represented by an ultra-local loworder (first or second order) differential equation. This model is input-output data-driven, i.e., continuously updated. It allows the synthesis of a simple controller of PID type, called intelligent-PID, or $i P I D$. This control strategy has already been successfully applied to quite different electric systems in [6], [7], [8]. Reference [9] exhibits a rather complete bibliography of the numerous concrete accomplishments of model-free control in the world. Some of them have been patented. As a matter of fact, model-free control yields important advantages for regulating complex systems including HVDCs such as: great conceptual simplicity, simplified parameterization, fast implementation and great robustness with respect to various disturbances.

In comparison to these previous applications, the case of the HVDC link treated here presents much faster dynamics dues to power electronic converters. The method is thus first tuned for this, especially for the estimation of the derivatives of measured signals. Next, it is tested in comparison with the classic vector control on a detailed VSC-HVDC system connected both sides to infinite buses modeled in Matlab/Simulink (Fig. 1).

The paper is structured as follows. The control problem is formulated in Section II. The basic principles of the modelfree control are briefly recalled in Section III. Implementation and validation scenarios and tests are discussed in Section IV. Robustness is analysed in Section V while Section VI is devoted to concluding remarks.

\section{SYSTEM DESCRIPTION}

The HVDC transmission link considered in our study in Fig. 1 is the detailed HVDC model provided as example (demonstration case) in SimPower Toolbox [10]. It consists of a 200 MVA (+/- $\mathrm{kV}$ DC) forced commutated VSC interconnection used to transmit power from a $230 \mathrm{kV}, 2000$ MVA, $50 \mathrm{~Hz}$ equivalent AC system to another identical one. These equivalent $\mathrm{AC}$ grids are modeled in a classic way 
by infinite buses $(E=1$ and $f=50 \mathrm{~Hz})$ and equivalent impedances $\left(L_{g_{i}}, r_{g_{i}}, i \in\{1,2\}\right)$ on each side of the HVDC. A circuit breaker is used to apply a three-phase to ground fault on the inverter $\mathrm{AC}$ side.

The discrete control system generates the three sinusoidal modulating signals that are the reference value of the bridge phase voltages. The power system and the control system are both discretized for a sample time Ts_power $=7.4010^{-6} \mathrm{~s}$ and Ts_control $=74.0610^{-6} s$ respectively (see [10] for more details on the model).

\section{Active Power Control}

The Active Power Control block in Fig. 2 is similar to the Reactive Power Control block. The extra ramping block ramps the power order towards the desired value with an adjusted rate when the control is active. The ramped value is reset to zero when the converter is blocked.

\section{MODEL-FreE CONTROL [4]}

For simplicity's sake let us restrict ourselves to SingleInput Single-Output systems. Instead of trying to write down a complex differential equation, introduce the ultra-local model

$$
\dot{y}=F+\alpha u,
$$

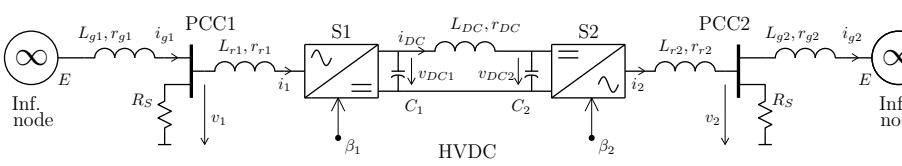

Figure 1. Benchmark for VSC-based HVDC in AC grid.

\section{A. Control structure}

The vector control is usually structured into two hierachic inner and outer loops which are also decoupled in time. The outer loops are slower and provide references to be tracked by the inner loops. This structure is used here for each converter as shown in Fig. 2. Convertor S1 controls the transmitted active power $P$ and the reactive power injection $Q_{1}$ at the left coupling point PCC1 in Fig. 1 while converter S2 controls the DC voltage $V_{D C_{2}}$ and reactive power injection $Q_{2}$ at the right coupling point $\mathrm{PCC} 2$.

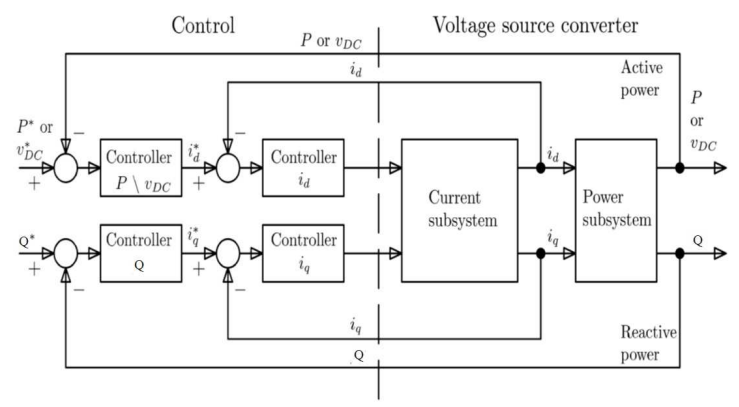

Figure 2. Vector-control of VSC-based HVDC.

\section{B. Reactive Power Control}

The Reactive Power Control regulator block in Fig. 2 combines an integral (I) control with a feed-forward control to increase the speed response. To avoid integrator wind-up the following actions are taken: the error is reset to zero, when the measured PCC voltage is less than a constant value (i.e., following a disturbance on the $\mathrm{AC}$ ); when the regulator output is limited, the limitation error is fed back with the right sign to the integrator input.

\section{where}

- $u$ and $y$ are, respectively, the input (control) and output variables,

- the derivation order of $y$ is 1 , like in most concrete situations,

- $\alpha \in \mathbb{R}$ is chosen by the designer such that $\alpha u$ and $\dot{y}$ are of the same magnitude.

The following explanations on $F$ might be useful:

- $F$ subsumes the knowledge of any model uncertainties and disturbances,

- $F$ is estimated via the measures of $u$ and $y$.

\section{A. Intelligent controllers}

The loop is closed by an intelligent proportional controller, or $i P$,

$$
u=-\frac{F-\dot{y}^{*}+K_{P} e}{\alpha}
$$

where

- $y^{\star}$ is the reference trajectory,

- $e=y-y^{\star}$ is the tracking error,

- $K_{P}$ is the usual tuning gain.

Combining equations (1) and (2) yields

$$
\dot{e}+K_{P} e=0,
$$

where $F$ does not appear anymore. Local exponential stability is ensured if $K_{P}>0$ :

- The gain $K_{P}$ is thus easily tuned.

- Robustness with respect to different types of disturbances and model uncertainties is achieved.

Remark 3.1: It is clear [4] that the gain tuning become much simpler than for classic PIs and PIDs ([11], [12]).

\section{B. Estimation of $F$}

For implementation purpose, $\mathrm{F}$ is defined by

$$
\tilde{F}=\dot{y}-\alpha u(t-h)
$$

where $u(t-h)$ is the control delayed by a small non-zero amount $h$ : it is the short time window needed for computations.

For the estimation of $\dot{y}$ algebraic numerical differentiation techniques ([13], [14]) are employed. These derivatives are 
replaced by integrals thanks to operational calculus [15]. Consider a signal $x(t)$ and its Taylor expansion at $t=0$

$$
x(t)=\sum_{i=1}^{+\infty} x^{(i)}(0) \frac{t^{i}}{i !} .
$$

A first-order truncation gives:

$$
x(t)=a_{0}+a_{1} t
$$

In order to estimate $a_{1}$, i.e., the first-order derivative, write it in the operational domain

$$
X(s)=\frac{a_{0}}{s}+\frac{a_{1}}{s^{2}} .
$$

In order to get rid of $a_{0}$, multiply both sides by $s$ and take the derivatives with respect to $s$ :

$$
-s^{-2} a_{1}=s \frac{d X(s)}{d s}+X(s) .
$$

Attenuate the corrupting noise by multiplying both sides by a negative power of $s .^{1}$

$$
-s^{-3} a_{1}=s^{-1} \frac{d X(s)}{d s}+s^{-2} X(s) .
$$

Remember [15] that $\frac{d}{d s}$ corresponds in the time domain to the multiplication by $-t$. It yields the following estimator $\tilde{a}_{1}$ of $a_{1}$ :

$$
\tilde{a}_{1}=\frac{3 !}{T^{3}} \int_{0}^{T}(T-2 \tau) y(\tau) d \tau,
$$

where the length $T$ of the time window may be quite small.

Remark 3.2: Estimation of $F$ may be directly derived [4] without the need of estimating the derivative of $y$ in Eq. (1).

\section{CONTROL IMPLEMENTATION AND VALIDATION}

The model-free control is implemented and tested in two stages:

1) Only the active power control loop is implemented at converter S1 with an iP in the hierarchical control structure presented in Fig. 2. The other control loops (for $Q_{1}, Q_{2}$ and $V_{D C_{2}}$ ) are not changed and thus maintained in the vector control approach as in the origin HVDC example of [10].

2) The whole control of converter $\mathrm{S} 1$ is synthesized with a multivariable (for $P$ and $Q_{1}$ control) model-free control.

Remark 4.1: The multivariable extension of Section III is straightforward (see [16], [17], and [5]).

\section{A. Test scenarios}

Basically the simulation runs from 0 to $4 \mathrm{~s}$. At $t=0 \mathrm{~s}$ the reference of the active power is subjected to a slope ramp of 0.43 . At $t=1.5 \mathrm{~s}, \mathrm{a}-0.1 \mathrm{pu}$ step is applied to the reference of active power (decrease from $1 p u$ to $0.9 \mathrm{pu}$ ). Steps are also applied to the reference of reactive power $Q_{2}$ (from 0 to $-0.1 p u$ ) at $t=2.0 \mathrm{~s}$. To quantify interactions with the other controls, a step on the reference of the DC voltage of converter S2 (decrease from 1pu to $0.95 p u$ ) is also considered

\footnotetext{
${ }^{1}$ Negative powers of $s$ correspond to integrals which are low-pass filters.
}

at $t=2.5 \mathrm{~s}$. Noise of $10^{-4} \mathrm{pu}$ is systematically added to measures.

The initial load-flow (steady-state) of the system corresponds to zero active power transit $(P=0)$, and zero reactive power injection $\left(Q_{1}=0, Q_{2}=0\right)$.

\section{B. Active power control}

1) Classic controller: Fig. 3 contains results with the classic vector control presented in Fig. 2. As mentioned in Section II, the $P$ loop consist in an intergral control. From the scenarios mentioned above, only the ramp starting at $t=0 \mathrm{~s}$ and the step of amplitude $-0.1 p u$ at $t=1.5$ are applied to the reference of active power at this stage.

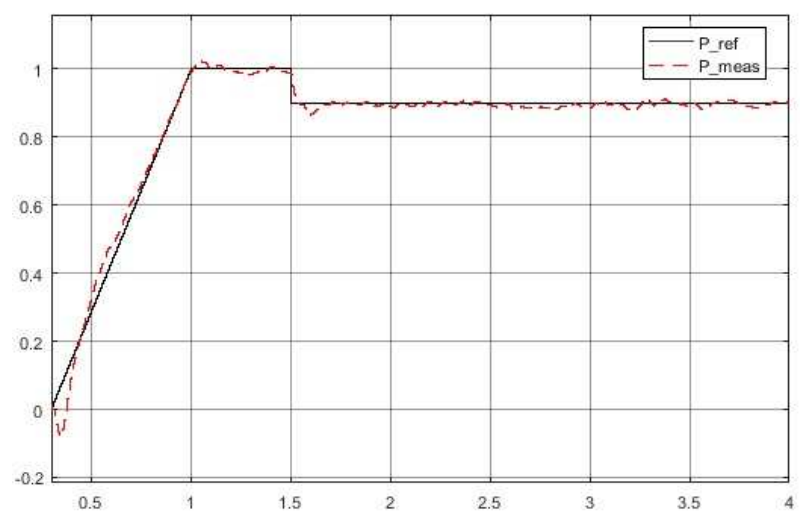

Figure 3. Active power response with classic vector controller.

2) Intelligent controller: An iP is now synthesized for the active power control loop. Indeed, with this choice, the modelfree control acts like a pure integrator through the coefficient $K_{P}$ in equation (2). The latter is chosen arbitrarily in such a way as to ensure desirable performance. The second setting parameter $(\alpha)$, it is chosen to adapt the control to the same order of magnitude as the first derivative of the measurement. The resulting control is given by

$$
u(k)=-\frac{\tilde{F}-\dot{P}^{*}+0.002 e}{5000}
$$

where $k$ denotes the $\mathrm{k}$-th sampling time and we select

$$
\tilde{F}=\dot{P}-5000 u(k-1)
$$

for estimating the active power derivative $\dot{P}$. To implement the latter derivative, we used the algebraic numerical differentiation of Section III with a number of samples $n=50$ and a sample time $T_{e}=5 \cdot 10^{-3} \mathrm{~s}$. It gives a window width of $T=0.25 \mathrm{~s}$. Figure 4 below represents the response of the resulting model-free control active power loop. Notice first that the tuning of parameters above provides responses at the same dynamics as the vector control loop (response to the step mentioned above in about $100 \mathrm{~ms}$ ). Performances are comparable for both controls. The noise level is also satisfactory in the model-free control loop. 


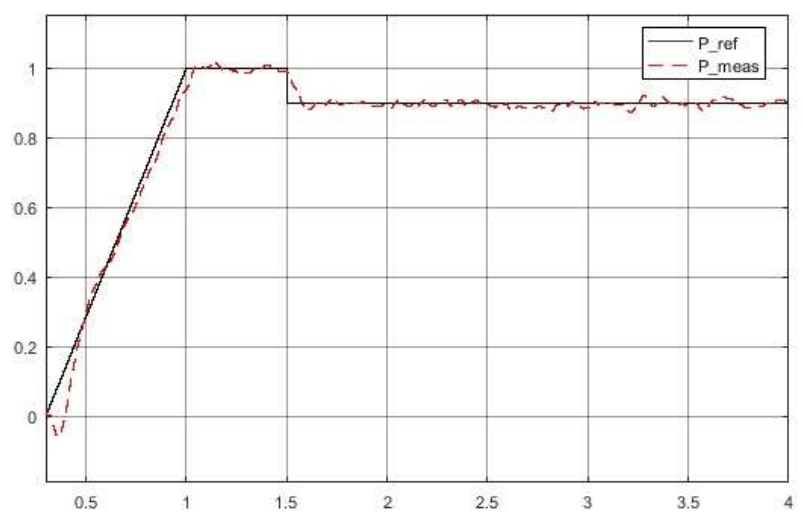

Figure 4. Active power response with an iP.

\section{Multivariable control of the active power and reactive power}

We study here the control of the two powers (active and reactive) simultaneously as we did before; first with the classic vector controller, next using the iP controller.

1) Classic vector controller: In addition to the ramp and the step on the active power reference used in the previous paragraph, a step of $-0.1 p u$ is now also applied on the reference of reactive power of converter $\mathrm{S} 1$ (from 0 to $-0.1 p u$ ) at $t=2.0 \mathrm{~s}$ to see the response of this loop also as well as the interaction with the $P$ loop (Fig. 5 and 6).

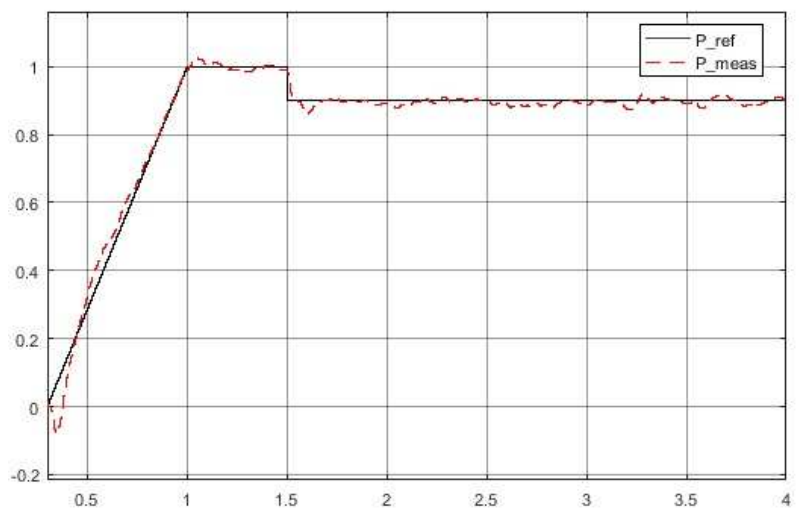

Figure 5. Active power response with a classic vector controller

2) Intelligent controller: Let $y_{1}^{*}$ and $y_{1}$ be the active power reference and active power measurement respectively and $y_{2}^{*}$ and $y_{2}$ the reactive power reference and reactive power measurement respectively. $u_{1}$ and $u_{2}$ represent the active power control and reactive power control respectively. Model (1) is now written in the multivariable form

$$
\left[\begin{array}{l}
\dot{P} \\
\dot{Q}
\end{array}\right]=\left[\begin{array}{l}
F_{1} \\
F_{2}
\end{array}\right]+\left[\begin{array}{cc}
\alpha_{1} & 0 \\
0 & \alpha_{2}
\end{array}\right]\left[\begin{array}{l}
u_{1} \\
u_{2}
\end{array}\right]
$$

The decoupling property, i.e., the diagonal character of the matrix $\alpha$ in Eq. (3), simplifies greatly the control synthesis.

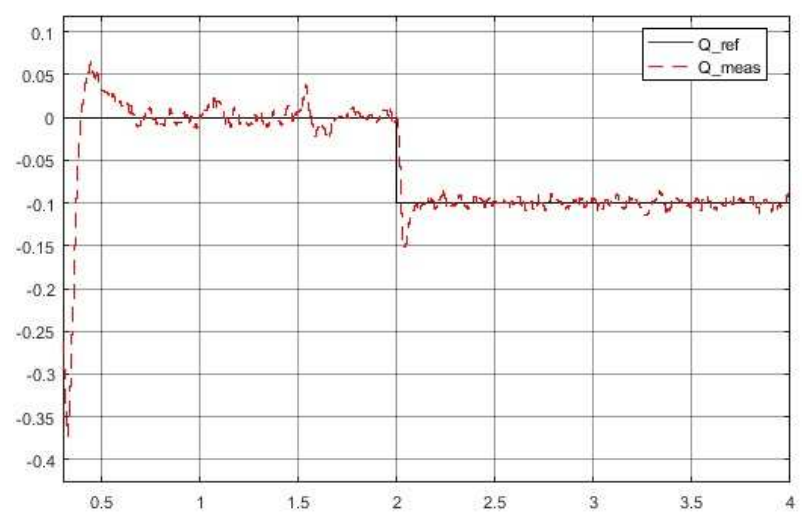

Figure 6. Reactive power response with a classic vector controller

Computer simulations below confirm this setting. ${ }^{2}$ Notice that $u_{1}$ et $u_{2}$ are defined below for $\alpha_{1}=\alpha_{2}=5000, K_{P_{1}}=0.002$ and $K_{P_{2}}=0.0006$

$$
\left[\begin{array}{l}
u_{1}(k) \\
u_{2}(k)
\end{array}\right]=\frac{-1}{5000}\left(\left[\begin{array}{c}
\tilde{F}_{1} \\
\tilde{F}_{2}
\end{array}\right]-\left[\begin{array}{l}
\dot{P}^{*} \\
\dot{Q}^{*}
\end{array}\right]+\left[\begin{array}{cc}
e_{1} & 0 \\
0 & e_{2}
\end{array}\right]\left[\begin{array}{c}
0.002 \\
0.0006
\end{array}\right]\right)
$$

As defined above

$$
\left[\begin{array}{c}
\tilde{F}_{1} \\
\tilde{F}_{2}
\end{array}\right]=\left[\begin{array}{l}
\dot{P} \\
\dot{Q}
\end{array}\right]-5000\left[\begin{array}{l}
u_{1}(k-1) \\
u_{2}(k-1)
\end{array}\right]
$$

For the estimation of the two derivatives, $\dot{y}_{1}$ and $\dot{y}_{2}$, the same algebraic numerical differentiation scheme as before has been employed, with the same parameters: number of samples $n=50$ and a sample time $T_{e}=510^{-3} s$. It yields a window width of $T=0.25 s$.

Responses in Fig. 7 and 8 show a good level of performance, i.e., a very low interaction between the two control loops. It is comparable with what has been achieved in the vector control setting. This is due to the fact that, even with this decoupled form of $\alpha$, information about the coupled dynamics is captured in the estimation of $F_{1}$ and $F_{2}$. Notice also that the vector control integrates a scheme of compensation of these interactions (see, e.g., [18]). The closed-loop provides also a good filtering of noise as in the case of the $P$ loop alone.

\section{Robustness Tests}

This section investigates the behavior of the controls in non nominal conditions.

${ }^{2}$ Until today, this decoupling property is valid in any concrete situation [16], [17]. 


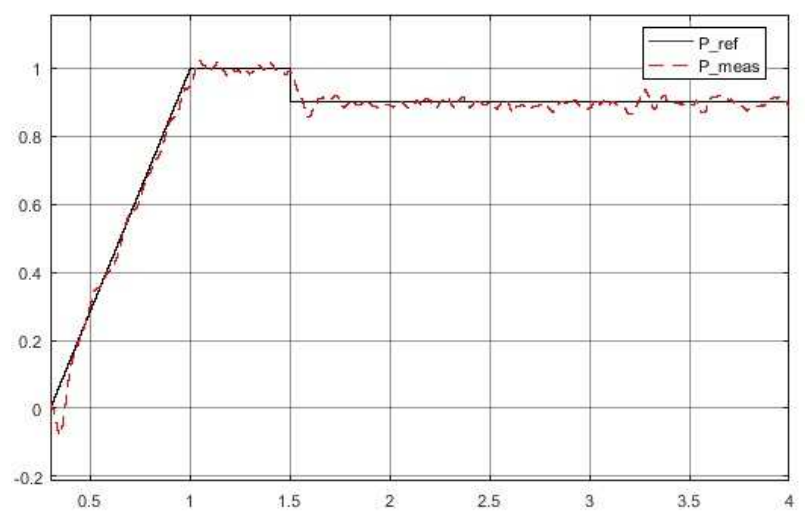

Figure 7. Active power response with a multivariable iP

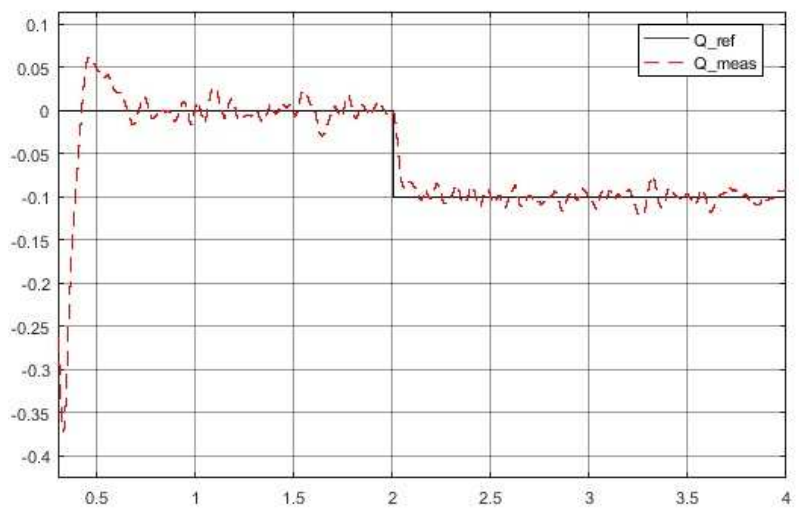

Figure 8. Reactive power response with a multivariable iP

\section{A. Grid variations}

First, grid variations like, e.g., line tripping, topology modifications, load/generation evolution are considered. On the simple benchmark in Fig. 1, this is implemented as variations of the equivalent impedance of the AC grid. $L_{g 1}$ and $r_{g 1}$ are varied (maintaining their ratio) from the operating point considered before for which $L_{g 1}=62.23 \times 10^{-3}$ in Figures 9 and 11 for the case of the classic vector controller and in Figures 10 and 12 using an iP. Notice that the controllers have not been recalibrated to take into account the grid variation. For average variations $\left(L_{g 1}=150 \times 10^{-3}\right)$ the responses are comparable but for large variations, the new controller has better robustness. As a matter of fact, $L_{g 1}=200 \times 10^{-3}$ corresponds to the stability limit of the classic vector control.

\section{B. Responses to large disturbances}

The behavior of the controls is now investigated in case of large grid disturbances which strongly excite nonlinear dynamics. Instead of the step on the reference of reactive power $Q$ of station $\mathrm{S} 1$, a $150 \mathrm{~ms}$ three-phase fault was applied at the PCC of station S1. Fig. 13 and 14 show that it is well rejected with both controllers.

\section{CONCLUSIONS}

The model-free control has been used to control a VSC HVDC transmission system. It has been shown that the

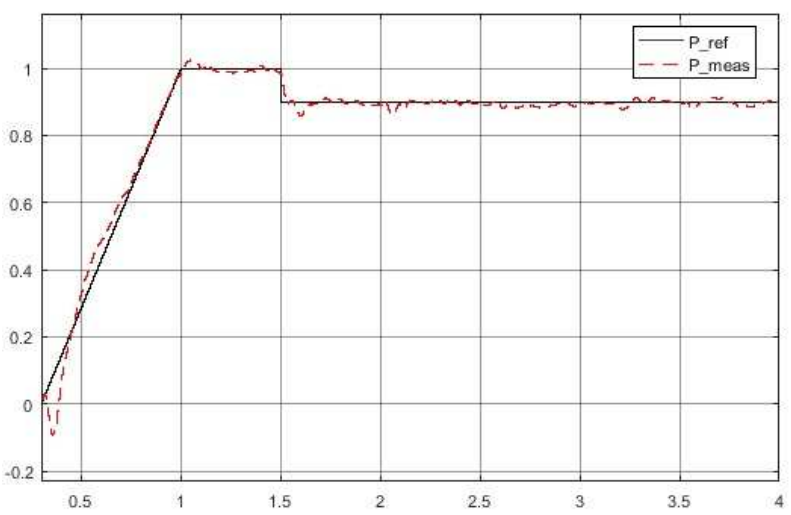

Figure 9. Active power response with classic vector controller $\left(L_{g 1}=150 \times\right.$ $\left.10^{-3}\right)$

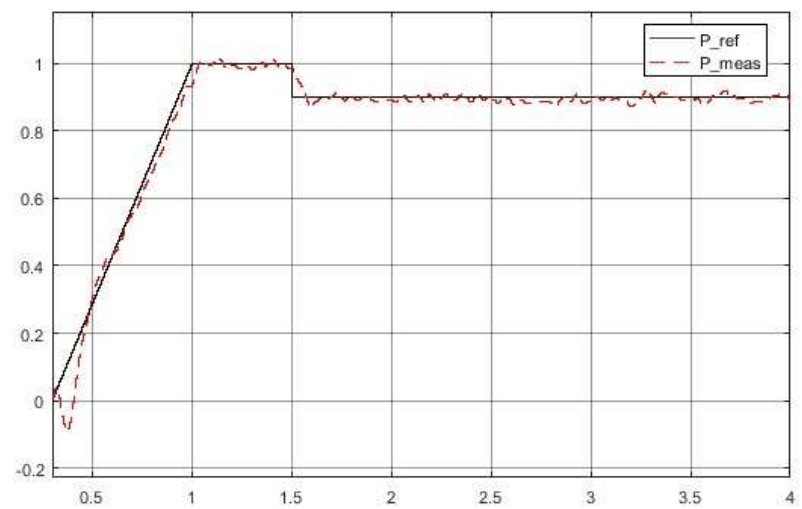

Figure 10. Active power response with a multivariable iP $\left(L_{g 1}=150 \times\right.$ $\left.10^{-3}\right)$

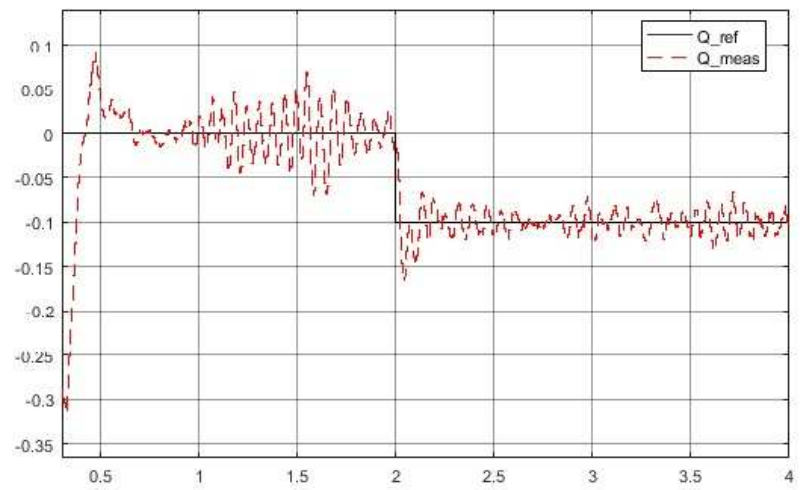

Figure 11. Reactive power response with classic vector controller $\left(L_{g 1}=\right.$ $200 \times 10^{-3}$ )

estimation of the derivatives of the output measures can be performed with good performances in the difficult context of the application: fast dynamics due to power electronics and noisy grid environment.

An iP is sufficient to ensure the same level of performances as the classic vector control and a great robustness with respect to the various disturbances. The advantage comes from its simple implementation which reduces to a simple proportional 


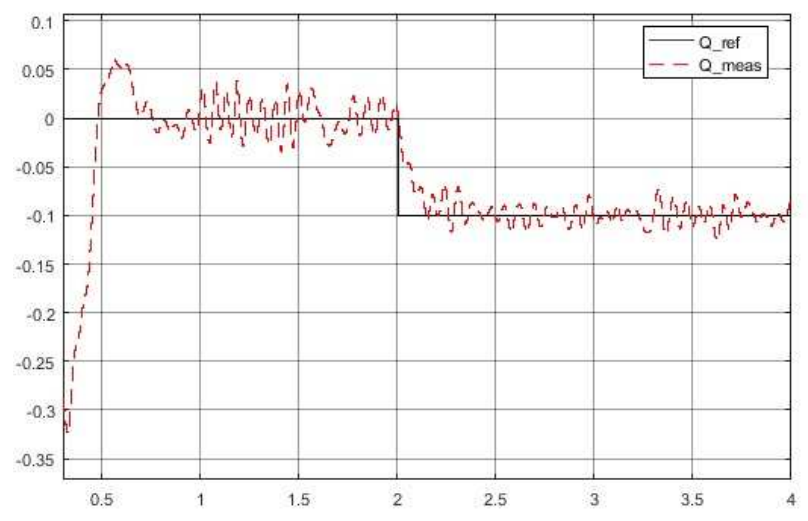

Figure 12. Reactive power response with a multivariable iP $\left(L_{g 1}=200 \times\right.$ $10^{-3}$ )

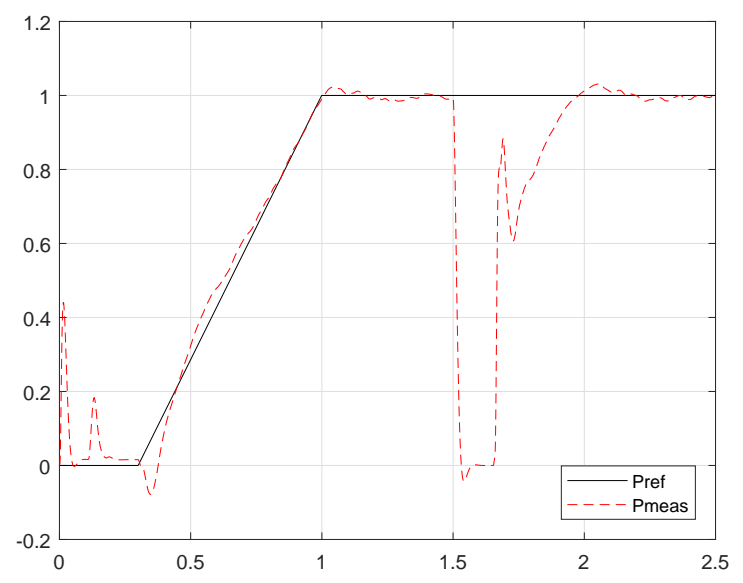

Figure 13. Active power response with a classic vector controller

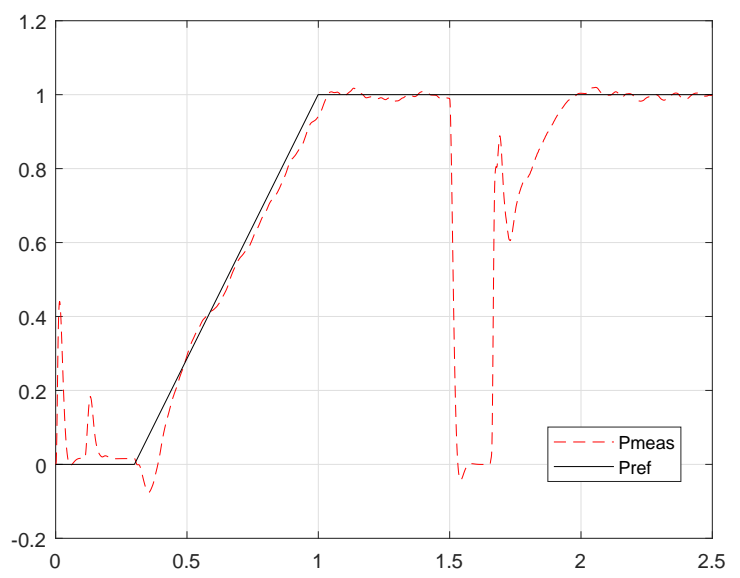

Figure 14. Active power response with an iP

gain setting by trial and error simulations. This is a major advantage in case of devices with complex dynamics like power electronics modules or grid (secondary) regulations which involve several dynamics elements of the power systems and for which it is difficult to obtain both accurate and small-dimension control models. Indeed, in these cases, it is interesting to promote a model-free solution.

A next step was done towards implementation of multiinput/multi-output intelligent control loops for one converter first and, next, for the whole HVDC link (the two converters and the DC part) and its validation in a large-scale grid context. This means to evaluate the impact of this new control on the dynamics of the AC neighbour zone of the HVDC. Thanks to Remark 3.2, it is expected that the model-free control provides better robustness against grid variations than the classic vector control.

After the full setting and validation on the HVDC case, this approach could be extended to the control of several grid devices based on power electronics like, e.g., renewable generators, energy storage, etc as an easy mean to take into account the grid environnement of the device.

\section{REFERENCES}

[1] L. Sigrist and F. Echavarren and L. Rouco and P. Panciatici, A fundamental study on the impact of HVDC lines on transient stability of power systems. 2015 IEEE PowerTech, Eindhoven, 2015.

[2] L. Arioua, B. Marinescu, Robust grid-oriented control of high voltage DC links embedded in an AC transmission system, Int. J. Robust Nonlin. Contr., 26, 1944-1961, 2016.

[3] L. Arioua, B. Marinescu, Multivariable control with grid objectives of an HVDC link embedded in a large-scale AC grid, Int. J. Electr. Power Energ. Syst., 72, 99-108, 2015.

[4] M. Fliess, C. Join, Model-free control, Int. J. Contr., 86, 2228-2252, 2013.

[5] M. Fliess, C. Join, Deux améliorations concurrentes des PID, ISTE OpenSci. Control, 2, 23 p., 2018. https://hal.archives-ouvertes.fr/hal-01687952/en/

[6] C. Join, G. Robert, M. Fliess, Vers une commande sans modèle pour aménagements hydroélectriques en cascade, $\sigma^{e}$ Conf. Internat. Francoph. Automat., Nancy, 2010. https://hal.archives-ouvertes.fr/inria-00460912/en/

[7] L. Michel, C. Join, M. Fliess, P. Sicard, A. Chériti, Model-free control of dc/dc converters, 12th IEEE Worksh. Contr. Model. Power Electron., Boulder, 2010. https://hal.archives-ouvertes.inria.fr/inria-00495776/en/

[8] O. Bara, M. Olama, S. Djouadi, T. Kuruganti, M. Fliess, C. Join, Modelfree load control for high penetration of solar photovoltaic generation. 49th North Amer. Power Symp., Morgantown, 2017. https://hal.archives-ouvertes.fr/hal-01558647/en/

[9] O. Bara, M. Fliess, C. Join, J. Day, S.M. Djouadi, Toward a model-free feedback control synthesis for treating acute inflammation. J. Theoret. Biology, 448, 26-37, 2018.

[10] VSC-Based HVDC Transmission System (Detailed Model) SimPowerSystems Toolbox, Simulink, Matlab-MathWorks, https://fr.mathworks.com/help/physmod/sps/powersys/ug/vsc-basedhvdc-link.html.

[11] K.J. Åström, R.M. Murray, Feedback Systems: An Introduction for Scientists and Engineers. Princeton University Press, 2008.

[12] A. O'Dwyer, Handbook of PI and PID Controller Tuning Rules (3rd ed.), Imperial College Press, 2009.

[13] M. Fliess, C. Join, H. Sira-Ramírez, Non-linear estimation is easy, Int. J. Model. Identif. Contr., 4, 2-27, 2008.

[14] M. Mboup, C. Join, M. Fliess, Numerical differentiation with annihilators in noisy environment, Numer. Algo., 50, 439-467, 2009.

[15] A. Erdélyi, Operational Calculus and Generalized Functions, Holt Rinehart Winston, 1962.

[16] F. Lafont, J.-F. Balmat, N. Pessel, M. Fliess, A model-free control strategy for an experimental greenhouse with an application to fault accommodation. Comput. Electron. Agricult., 110, 139-149, 2015.

[17] L. Menhour, B. d'Andréa-Novel, M. Fliess, D. Gruyer, H. Mounier, An efficient model-free setting for longitudinal and lateral vehicle control. Validation through the interconnected pro-SiVIC/RTMaps prototyping platform. IEEE Trans. Intel. Transport. Syst., 19, 461-475, 2018.

[18] W. Leonhard, Control of Electrical Drives, Springer 1996. 